\title{
Impact of explained $v$. unexplained front-of-package nutrition labels on parent and child food choices: a randomized trial
}

\author{
Dan J Graham ${ }^{1,2, *}$, Rachel G Lucas-Thompson ${ }^{3}$, Megan P Mueller ${ }^{2,4}$, Melanie Jaeb² and \\ Lisa Harnack ${ }^{2}$ \\ ${ }^{1}$ Colorado School of Public Health and Department of Psychology, Colorado State University, 1876 Campus \\ Delivery, Fort Collins, CO 80523, USA: ${ }^{2}$ Division of Epidemiology and Community Health, University of Minnesota, \\ Minneapolis, MN, USA: ${ }^{3}$ Colorado School of Public Health and Department of Human Development and Family \\ Studies, Colorado State University, Fort Collins, CO, USA: ${ }^{4}$ Friedman School of Nutrition Science and Policy, \\ Tufts University, Boston, MA, USA
}

Submitted 16 February 2016: Final revision received 24 August 2016: Accepted 1 September 2016: First published online 17 0ctober 2016

\begin{abstract}
Objective: The present study investigated whether parent/child pairs would select more healthful foods when: (i) products were labelled with front-of-package (FOP) nutrition labels relative to packages without labels; (ii) products were labelled with colour-coded Multiple Traffic Light (MTL) FOP labels relative to monochromatic Facts up Front (FuF) FOP labels; and (iii) FOP labels were explained via in-aisle signage $v$. unexplained.

Design: Participants were randomly assigned to one of five conditions: (i) FuF labels with in-aisle signs explaining the labels; (ii) FuF labels, no signage; (iii) MTL labels with in-aisle signage; (iv) MTL labels, no signage; (v) control group, no labels/signage. Saturated fat, sodium, sugar and energy (calorie) content were compared across conditions.

Setting: The study took place in a laboratory grocery aisle.

Subjects: Parent/child pairs ( $n$ 153) completed the study.

Results: Results did not support the hypothesis that MTL labels would lead to more healthful choices than FuF labels. The presence of FOP labels did little to improve the healthfulness of selected foods, with few exceptions (participants with $v$. without access to FOP labels selected lower-calorie cereals, participants with access to both FOP labels and in-aisle explanatory signage selected products with less saturated fat $v$. participants without explanatory signage).

Conclusions: Neither MTL nor FuF FOP labels led to food choices with significantly lower saturated fat, sodium or sugar. In-aisle signs explaining the FOP labels were somewhat helpful to consumers in making more healthful dietary decisions. New FOP labelling programmes could benefit from campaigns to increase consumer awareness and understanding of the labels.
\end{abstract}

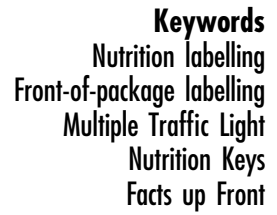

The Dietary Guidelines for Americans and the WHO recommend limiting intakes of sodium, added sugars, saturated fat and total energy for health promotion and disease prevention ${ }^{(1,2)}$. Diet-related diseases represent a considerable global public health threat, projected to incur costs of millions of lives and billions of dollars by $2030^{(3-5)}$. The US Food and Drug Administration has mandated that pre-packaged foods in the USA carry Nutrition Facts Labels $(\mathrm{NFL})^{(6)}$ to help 'consumers make informed choices and maintain healthy dietary practices, ${ }^{\text {(7) }}$. Similar nutrition information panels are mandated in many countries (i.e. Australia, Canada, the European Union, Hong Kong, India, Malaysia, New Zealand) ${ }^{(8)}$. Informed food purchasing is a global priority, but unfortunately consumers pay little attention to $\mathrm{NFL}^{(9)}$, likely due to their location on the side facing of food packages ${ }^{(10)}$. In addition, consumer understanding of NFL is low, especially for children and low-health-literate adults ${ }^{(11-13)}$. It is difficult for these demographic groups to understand and effectively use NFL because of the large amount of information presented and because NFL lack interpretation aids (e.g. one must know that a high percentage daily value (\%DV) is desirable for some nutrients like calcium whereas a low $\% \mathrm{DV}$ is the goal for others like sodium) ${ }^{(14)}$.

There is growing consensus that NFL alone are inadequate for guiding consumers, particularly children, 
towards more healthful choices; even the Food and Drug Administration, which mandated NFL, acknowledges the challenges with using and understanding them ${ }^{(15)}$. Policy makers have made providing more useful nutrition information a priority ${ }^{(16)}$. Towards this end, the Food and Drug Administration has solicited input (e.g. research results) on how to improve nutrition labelling and plans to implement front-of-package (FOP) labelling as part of this initiative $^{(16)}$

FOP labels have the potential to address many NFL shortcomings. The prominent location of FOP labels increases consumer attention relative to $\mathrm{NFL}^{(10)}$. FOP labels are widely used by consumers when available ${ }^{(17)}$ and, due to their translation of raw numbers into healthfulness metrics (e.g. stars, colours), they can be more easily interpreted by adults and children than can NFL. The use of all types of nutrition labelling (e.g. NFL, energy labelling on menus) appears strongly driven by consumer motivation $^{(18)}$, in that only those who are motivated to consume healthy foods use available labels to pursue healthier options. However, given FOP labels' prominence and amenability to heuristic processing, even those with lower levels of motivation may by influenced by FOP labels when selecting foods. It is nevertheless important to consider participant motivation as a moderator of the effects of FOP label availability on the healthfulness of food selection.

Data are lacking about consumer use of FOP labels to inform food choice ${ }^{(17)}$. In particular, as noted in a recent review of research needs on FOP labelling ${ }^{(19)}$, there are major knowledge gaps about how FOP labels are used by consumers and whether FOP labels meet the needs of consumers with lower levels of literacy and numeracy. In addition, it is unclear whether FOP labels alone are sufficient to prompt healthier food choices or whether additional support is needed to inform consumers that these labels are available, to guide interpretation of the labels and/or to encourage consumers to use these labels to make healthier food choices. Recent work suggests that FOP labels alone are less effective in reaching consumers than FOP labels that are highlighted with in-aisle signage. Specifically, 95\% of consumers viewed FOP labels during a shopping task when these labels were accompanied by explanatory in-aisle signage, while only $27 \%$ of consumers viewed the same labels when no such signage was provided $^{(10)}$.

The present analysis examines the impact of FOP labels and in-aisle signage identifying and explaining those labels on the healthfulness of foods selected by consumers in a randomized, controlled experiment. Two FOP label formats were tested. The first was an FOP system introduced as Nutrition Keys by the US Grocery Manufacturers Association and Food Marketing Institute in January $2011^{(20)}$ and later renamed Facts up Front (FuF); these labels use a monochromatic background (Fig. 1) and four differentiated sections to convey the amount per serving of
Public health experts recommend that Americans consume:

Less than 2000 calories per day

Less than $20 \mathrm{~g}$ saturated fat per day

Less than $2400 \mathrm{mg}$ sodium per day

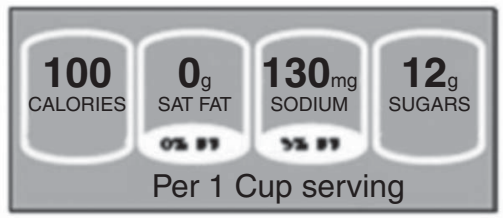

High Calories

Sat. fat.

Sodium

Sugars $=$

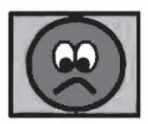

What do the traffic light colours mean?

RED means the food is high in something we should eat less of (like fat or sodium)

AMBER means that food has a medium amount of something we should eat less of (like fat or sodium).

GREEN means the food is low in something we should eat less of (like fat or sodium).

Healthier choices have more greens and fewer reds!
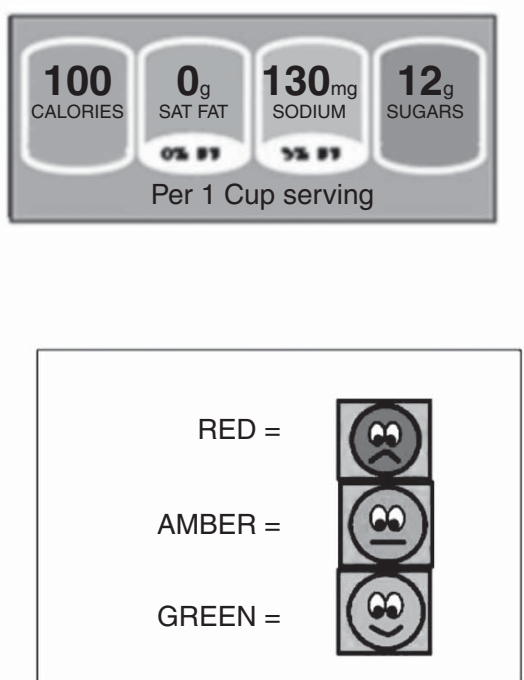

Fig. 1 Facts up Front (FuF; top) and Multiple Traffic Light (MTL; bottom) front-of-package (FOP) labels, and their respective in-aisle explanatory signage 
energy (calories), saturated fat, sodium and sugar. At the manufacturer's discretion, FuF labels can also include up to two additional 'nutrients to promote ${ }^{,(20)}$. In the present study, only the four required nutrients to limit were included. The second FOP label type was the Multiple Traffic Light (MTL), which uses colour designations to indicate low (green), medium (amber) and high (red) levels of key 'nutrients to limit'. For the present study, MTL labels used the same label format as the FuF labels (Fig. 1), with the colour coding applied to the saturated fat, sodium and sugars sections, based on the criteria used in the UK's MTL labelling system ${ }^{(21)}$.

FuF labels were included in the study because they have been widely adopted in the USA, but no data have yet been published on their impact on consumer food choices. It will be useful for informing the Food and Drug Administration about whether the labels already in place are helping consumers to make more healthful choices, or whether an alternative system would better serve this purpose. The alternative system tested here, MTL labelling, is the FOP system with the most empirical research supporting its use ${ }^{(17,22)}$ and is hypothesized to lead to more healthful food choices than FuF labels, as the MTL's colour coding provides added information beyond the FuF's numeric data. Indeed, UK consumers strongly prefer to avoid foods labelled with red lights ${ }^{(23)}$. Traffic light information is presented in an easily and quickly understood format that can be useful to consumers without requiring in-depth processing of the label's numeric content. Previous European and Australian research has demonstrated that consumers are better able to answer health-related questions about food products when the products are labelled with traffic light systems relative to monochromatic systems like FuF labels ${ }^{(24,25)}$. US consumers more successfully compared foods' healthfulness and estimated nutrient levels when using MTL $v$. FuF labels ${ }^{(26)}$.

The added value of in-aisle signage (Fig. 1) was also tested in the current study. Signage was of interest for several reasons. Previous research suggests that it is helpful to provide consumers with interpretational aids for comparing the contributions that individual foods make towards fulfilling daily requirements ${ }^{(14,27)}$. MTL colours provide this context, but American consumers were expected to be unfamiliar with MTL labels because they are not yet commonly used in the USA; therefore MTL signage explained the meaning of the green, amber and red designations. The potential for American consumers to be initially confused by MTL labelling is supported by consumer misunderstanding of the meaning of MTL labels in the UK prior to the widespread adoption of MTL labelling ${ }^{(28)}$. Because there were no colours to provide context regarding low, medium and high nutrient levels for FuF participants, contextual information was included on the FuF signage via the public health recommendations used to calculate \%DV.
Finally, signage was designed to help make the FOP labels useful for individuals with low literacy, including children; face icons were used to achieve this goal. For MTL labels, smiling, neutral and frowning faces corresponded to low, medium and high levels of the nutrients to limit, respectively. The FuF label format was less conducive to providing symbolic information to children due to the lack of differentiating colours; the corresponding portion of the FuF signage paired the frowning face with high levels of each FuF label component.

It was hypothesized that signage would increase healthfulness of food choices because using nutrition labels is linked with healthier food choices ${ }^{(17)}$ and signage was expected to increase label use for several reasons. Signage would: (i) notify previously unaware consumers of the FOP labels' presence; (ii) explain how to interpret label contents; and (iii) help individuals with low literacy, including children, identify healthy options.

In summary, the present study tested the impact of two FOP labels on the nutrient composition of foods chosen by consumers in a controlled experiment. It was hypothesized that participants with access to FOP labels would make healthier food choices (defined as items lower in at least one of the nutrients to limit highlighted on the FOP label) than participants without FOP labels, particularly if they reported high levels of motivation to eat healthful foods. Further, it was anticipated that participants with access to MTL labels would make more healthful food choices than those with access to FuF labels. Finally, it was expected that in-aisle signage explaining FOP labels would lead participants to select more healthful foods than participants without signage.

\section{Methods}

\section{Participants}

Participants were recruited for a study of 'family food preferences' through print, online and in-person sources. Parents were eligible to participate if they could read English, had a 6-9- year-old child and could attend a $1 \mathrm{~h}$ laboratory visit during which they did not wear eyeglasses, as the study required participants to wear eye-tracking glasses. One hundred and fifty-three parent/child pairs from Minneapolis/St. Paul, MN, USA, enrolled. Parents were primarily female, white and non-Hispanic, and married. Mean parent BMI was $27.9 \mathrm{~kg} / \mathrm{m}^{2}$ and parents represented families with a range of educational and socio-economic backgrounds (Table 1).

\section{Procedures}

Participants attended a one-time, $90 \mathrm{~min}$ visit at the University of Minnesota in 2012 or 2013. Before beginning the study procedures, all participants provided written 
Table 1 Demographic characteristics of parents and children, for the full sample and by experimental condition; parent/child pairs from Minneapolis/St. Paul, MN, USA, 2012-2013

\begin{tabular}{|c|c|c|c|c|c|c|c|c|c|c|c|c|}
\hline & \multirow{2}{*}{\multicolumn{2}{|c|}{$\begin{array}{c}\text { Full sample } \\
\text { ( } n \text { 153) }\end{array}$}} & \multicolumn{10}{|c|}{ Experimental condition* } \\
\hline & & & \multicolumn{2}{|c|}{$1(n 33)$} & \multicolumn{2}{|c|}{$2(n 29)$} & \multicolumn{2}{|c|}{$3(n 26)$} & \multicolumn{2}{|c|}{$4(n 35)$} & \multicolumn{2}{|c|}{$5(n 30)$} \\
\hline & $n$ & $\%$ & $n$ & $\%$ & $n$ & $\%$ & $n$ & $\%$ & $n$ & $\%$ & $n$ & $\%$ \\
\hline \multicolumn{13}{|l|}{ Family characteristics } \\
\hline \multicolumn{13}{|l|}{ Race/ethnicity } \\
\hline White & 126 & $82 \cdot 4$ & 27 & $81 \cdot 8$ & 23 & $79 \cdot 3$ & 22 & $84 \cdot 6$ & 27 & $77 \cdot 1$ & 27 & $90 \cdot 0$ \\
\hline Black & 12 & $7 \cdot 8$ & 3 & $9 \cdot 1$ & 2 & 6.9 & 2 & $7 \cdot 7$ & 3 & 8.6 & 2 & 6.7 \\
\hline Native American & 11 & $7 \cdot 2$ & 2 & $6 \cdot 1$ & 3 & $10 \cdot 3$ & 1 & $3 \cdot 8$ & 2 & $5 \cdot 7$ & 3 & $10 \cdot 0$ \\
\hline Other & 9 & 5.9 & 1 & 3.0 & 1 & 3.4 & 2 & $7 \cdot 7$ & 3 & $8 \cdot 6$ & 2 & $6 \cdot 7$ \\
\hline \multicolumn{13}{|l|}{ Family income } \\
\hline$\leq \$ \cup S 25000$ & 19 & $12 \cdot 4$ & 2 & $6 \cdot 1$ & 4 & 13.8 & 0 & 0.0 & 8 & $22 \cdot 9$ & 5 & $16 \cdot 7$ \\
\hline \$US $25001-50000$ & 28 & $18 \cdot 3$ & 7 & 21.2 & 6 & $20 \cdot 7$ & 7 & $26 \cdot 9$ & 3 & 8.6 & 5 & $16 \cdot 7$ \\
\hline \$US $50001-75000$ & 35 & $22 \cdot 9$ & 5 & $15 \cdot 2$ & 7 & $24 \cdot 1$ & 6 & 23.1 & 9 & $25 \cdot 7$ & 8 & $26 \cdot 7$ \\
\hline \$US $75001-100000$ & 36 & 23.5 & 9 & $27 \cdot 3$ & 7 & $24 \cdot 1$ & 9 & $34 \cdot 6$ & 7 & $20 \cdot 0$ & 4 & $13 \cdot 3$ \\
\hline \$US $100001-125000$ & 12 & $7 \cdot 8$ & 1 & 3.0 & 2 & 6.9 & 2 & $7 \cdot 7$ & 4 & 11.4 & 3 & $10 \cdot 0$ \\
\hline$\geq \$ \cup S 125001$ & 18 & 11.8 & 8 & $24 \cdot 2$ & 2 & 6.9 & 1 & $3 \cdot 8$ & 2 & $5 \cdot 7$ & 5 & $16 \cdot 7$ \\
\hline \multicolumn{13}{|l|}{ Parent characteristics } \\
\hline Age (years), mean and SD & $38 \cdot 1$ & $6 \cdot 2$ & 37.4 & $4 \cdot 7$ & $39 \cdot 3$ & $6 \cdot 9$ & $38 \cdot 8$ & $6 \cdot 0$ & $37 \cdot 5$ & $7 \cdot 6$ & 38.0 & $5 \cdot 1$ \\
\hline Sex (female) & 134 & 87.6 & 32 & 97 & 23 & $79 \cdot 3$ & 22 & 84.6 & 29 & 82.9 & 28 & 93.3 \\
\hline \multicolumn{13}{|l|}{$\mathrm{BMI}$} \\
\hline$<18.50 \mathrm{~kg} / \mathrm{m}^{2}$ & 2 & $1 \cdot 3$ & 1 & 3.0 & 0 & 0.0 & 0 & 0.0 & 0 & 0.0 & 1 & $3 \cdot 3$ \\
\hline $18.50-24.99 \mathrm{~kg} / \mathrm{m}^{2}$ & 56 & $36 \cdot 6$ & 12 & $36 \cdot 4$ & 10 & 34.5 & 7 & $26 \cdot 9$ & 15 & 42.9 & 12 & 40 \\
\hline $25.00-29.99 \mathrm{~kg} / \mathrm{m}^{2}$ & 50 & $32 \cdot 7$ & 15 & 45.5 & 8 & 27.6 & 9 & $34 \cdot 6$ & 10 & 28.6 & 8 & $26 \cdot 7$ \\
\hline$\geq 30.00 \mathrm{~kg} / \mathrm{m}^{2}$ & 45 & $29 \cdot 4$ & 5 & $15 \cdot 2$ & 11 & 37.9 & 10 & 38.5 & 10 & 28.6 & 9 & $30 \cdot 0$ \\
\hline \multicolumn{13}{|l|}{ Marital status } \\
\hline Never married & 19 & $12 \cdot 4$ & 6 & $18 \cdot 2$ & 4 & 13.8 & 0 & 0.0 & 5 & $14 \cdot 3$ & 4 & $13 \cdot 3$ \\
\hline Married & 112 & 73.2 & 25 & $75 \cdot 8$ & 19 & 65.5 & 21 & $80 \cdot 8$ & 23 & 65.7 & 24 & $80 \cdot 0$ \\
\hline Other & 16 & 10.5 & 1 & 3.0 & 5 & $17 \cdot 2$ & 4 & $15 \cdot 4$ & 4 & 11.4 & 2 & 6.7 \\
\hline \multicolumn{13}{|l|}{ Education } \\
\hline Less than college & 14 & $9 \cdot 2$ & 1 & 3.0 & 3 & $10 \cdot 3$ & 2 & $7 \cdot 7$ & 5 & $14 \cdot 3$ & 3 & $10 \cdot 0$ \\
\hline Some college $(<4$ years) & 48 & 31.4 & 6 & $18 \cdot 2$ & 11 & 37.9 & 11 & $42 \cdot 3$ & 11 & 31.4 & 9 & 30.0 \\
\hline College degree (4 years) & 55 & 35.9 & 15 & 45.5 & 9 & 31.0 & 10 & 38.5 & 8 & 22.9 & 13 & 43.3 \\
\hline Graduate or professional education & 31 & $20 \cdot 3$ & 10 & 30.3 & 5 & $17 \cdot 2$ & 2 & 7.7 & 9 & $25 \cdot 7$ & 5 & $16 \cdot 7$ \\
\hline \multicolumn{13}{|l|}{ Healthy food purchasing } \\
\hline Not important & 0 & 0.0 & 0 & 0.0 & 0 & 0.0 & 0 & 0.0 & 0 & 0.0 & 0 & 0.0 \\
\hline Somewhat important & 34 & $22 \cdot 2$ & 7 & $21 \cdot 2$ & 5 & $17 \cdot 2$ & 7 & $26 \cdot 9$ & 8 & 22.9 & 7 & 23.3 \\
\hline Important & 58 & 37.9 & 12 & $36 \cdot 4$ & 7 & $24 \cdot 1$ & 10 & 38.5 & 14 & $40 \cdot 0$ & 15 & $50 \cdot 0$ \\
\hline Very important & 54 & $35 \cdot 3$ & 13 & 39.4 & 14 & $48 \cdot 3$ & 8 & $30 \cdot 8$ & 11 & 31.4 & 8 & $26 \cdot 7$ \\
\hline \multicolumn{13}{|l|}{ Child characteristics } \\
\hline Age (years), mean and SD & $7 \cdot 3$ & $1 \cdot 1$ & $7 \cdot 3$ & $1 \cdot 1$ & $7 \cdot 6$ & 1.0 & $7 \cdot 4$ & $1 \cdot 2$ & 7.3 & 1.0 & $7 \cdot 2$ & 1.2 \\
\hline Sex (female) & 75 & 49.0 & 16 & 48.5 & 16 & $55 \cdot 2$ & 15 & $57 \cdot \overline{7}$ & 15 & 42.9 & 13 & 43.3 \\
\hline \multicolumn{13}{|l|}{ BMl } \\
\hline$>85$ th percentile & 38 & 24.8 & 10 & $30 \cdot 3$ & 8 & $27 \cdot 6$ & 6 & 23.1 & 6 & $17 \cdot 1$ & 8 & $26 \cdot 7$ \\
\hline$>95$ th percentile & 11 & $7 \cdot 2$ & 1 & 3.0 & 4 & 13.8 & 1 & 3.8 & 4 & 11.4 & 1 & $3 \cdot 3$ \\
\hline$>97$ th percentile & 8 & 5.2 & 0 & 0.0 & 3 & $10 \cdot 3$ & 1 & 3.8 & 3 & 8.6 & 1 & 3.3 \\
\hline
\end{tabular}

For some variables, sums do not equal $100 \%$ due to non-responding (e.g. income) and/or selecting multiple responses (e.g. race/ethnicity).

${ }^{*}$ Conditions: 1, Facts up Front (FuF) labels, no signage; 2, FuF labels with signage; 3, Multiple Traffic Light (MTL) labels, no signage; 4, MTL labels with signage;

5 , control, no labels or signage.

informed consent (parents) and assent (children). All study procedures were approved by the University of Minnesota's Institutional Review Board. Study aims and primary outcomes were defined $a$ priori and recorded in advance of data collection and analysis ${ }^{(29)}$. Participants were compensated with six foods of their choosing, a \$US 20 gift card, parking remuneration and a small toy.

Using a 2 (FOP label type: FuF, MTL) $\times 2$ (explanatory signage: present, absent) + 1 (control group: no FOP labels, no signage) design, participants were randomly assigned to one of five conditions (see Fig. 2 for diagram of study flow). Randomization to condition was blocked by day, so the aisle was reset with the ninety foods appropriate to the condition only once per day. Randomization was conducted by the project manager via random number draw. Parent and child participants were weighed and measured by research staff using a standard, calibrated scale and stadiometer before beginning the food selection task in a separate room.

Participants wore eye-tracking glasses (Tobii Technology, Danderyd, Sweden) during the food selection task to objectively measure attention to FOP labels. The data reflecting parental attention to nutrition information and parent/child interactions during the food choice task are 


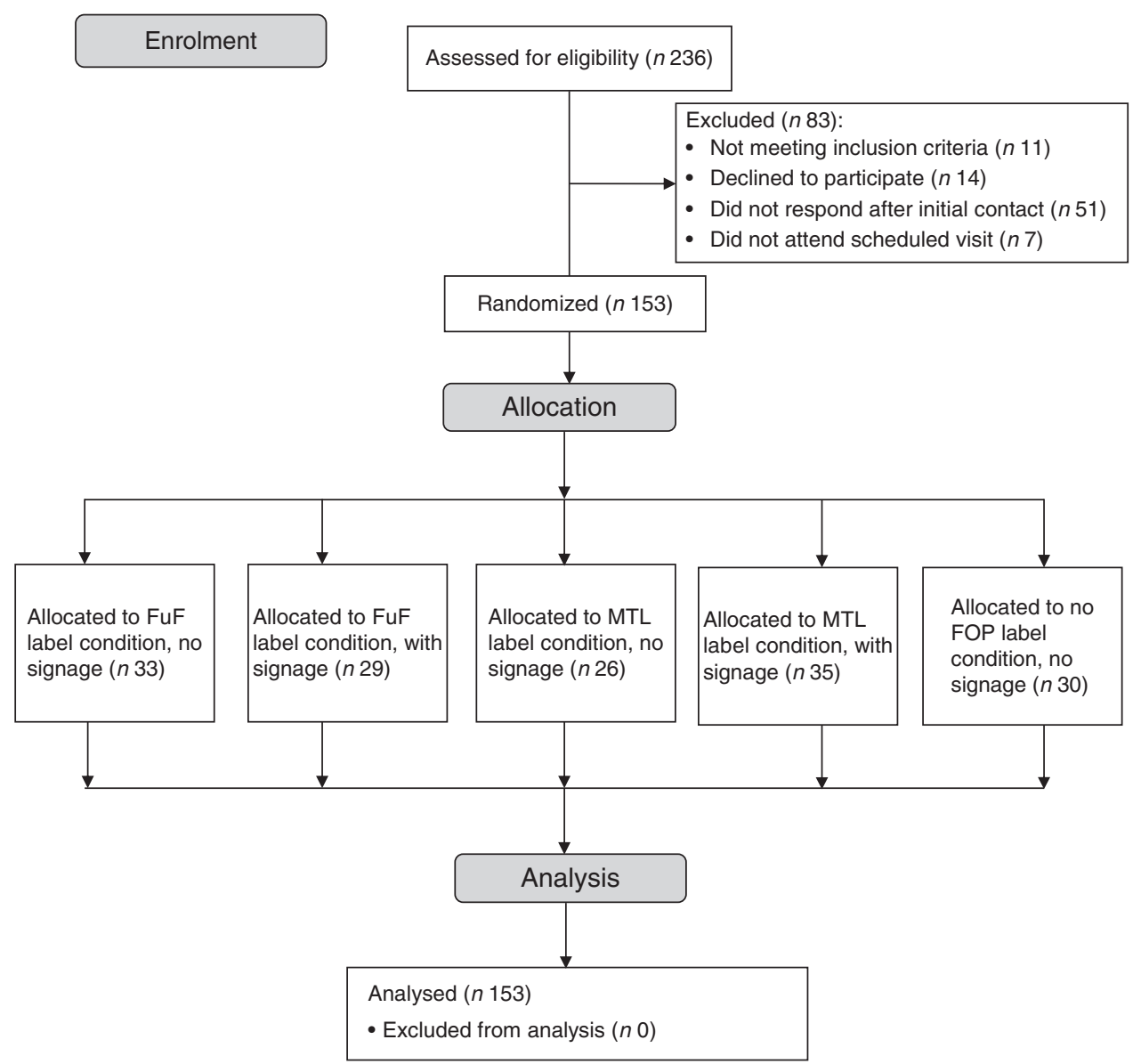

Fig. 2 Study enrolment and randomization flow diagram (FuF, Facts up Front; MTL, Multiple Traffic Light; FOP, front-of-package)

reported elsewhere ${ }^{(10)}$ (RG Lucas-Thompson, DJ Graham, D McPhee et al., unpublished results). Prior to entering the laboratory grocery aisle, participants were given instructions about the food selection task. Participants were told to behave as they typically would when food shopping and work together to select two foods from each of three categories ('crackers/cookies', 'cereals' and 'chips/snacks', for a total of six foods) to take home. To reduce their potential influence on participants' food choices, researchers remained outside the room while participants selected foods and only one participant pair was in the aisle at a time.

The grocery aisle contained ninety foods, thirty each across three food categories selected for relatively long shelf-life, pre-packaged status (i.e. they would be impacted by labelling initiatives), within-category variety on the nutrients of interest (i.e. the amounts of saturated fat, sodium and sugar in the products within each category were variable) and common occurrence in supermarkets and convenience stores. These three categories were: (i) crackers/cookies (e.g. cheese crackers, wheat crackers, vanilla cookies, chocolate cookies); (ii) breakfast cereals (e.g. puffed rice, shredded wheat); and (iii) chips/snacks (e.g. potato chips, tortilla chips, pretzels, popcorn).
Products were displayed in their actual packages and were positioned in the same relative locations as in the grocery store from which they were purchased (e.g. cereals marketed towards children were located on shelves closer to child eye-level than cereals marketed towards adults). Price tags were affixed to shelves below each product and displayed the cost of the items when purchased in the local grocery store when the study began in May 2012 (prices for cereals ranged from \$US 3.39 to \$US 4.94; chips/snacks ranged from \$US 2.09 to \$US 5.09; crackers/ cookies ranged from \$US 1.69 to \$US 4.79). Three sets of products were used for the experiment, one for each label condition (MTL, FuF, no label). For the MTL and FuF conditions, $5 \mathrm{~cm} \times 2.5 \mathrm{~cm}$ FOP labels were affixed to the food packages in the upper right-hand corner of the package. The in-aisle signage (Fig. 1) included four signs of approximately $13 \mathrm{~cm} \times 18 \mathrm{~cm}$. Two signs were positioned approximately one-third of the distance from each end of the aisle (one at a height chosen to approximate eye-level for children and one at a height intended to be readily visible to adults).

Participants could pick up and examine as many products as they wished when making their choices. When their decisions were finalized, they were asked to 
place into a grocery basket two foods from each of the three categories, for a total of six foods that they then took home with them upon study completion. After selecting the foods, parents completed a questionnaire in which they self-reported demographic information including race, ethnicity, family income, age, sex, marital status, education, as well as their motivation for eating healthfully, which was assessed with the following item: 'How important is it to you to purchase healthy foods?' Answer choices were: 'Not important', 'Somewhat important', 'Important' and 'Very important'. While parents completed the questionnaire, study staff bagged and recorded (for subsequent nutritional assessment) the six food items that participants had placed in their grocery basket.

\section{Measures}

Food healthfulness was quantified as the average amount per serving of calories, saturated fat, sodium and sugar of the six chosen products, with lower levels of these nutrients considered more healthful. BMI was calculated $\left(\mathrm{kg} / \mathrm{m}^{2}\right)$ based on measured height and weight. Parents self-reported their age, race, ethnicity and sex.

\section{Statistical analysis}

ANOVA were used to compare amounts of saturated fat, sodium, sugar and calories across all six foods chosen and within the three food categories (crackers/cookies, cereals, chips/snacks) across the appropriate combinations of study conditions to test each hypothesis. For Hypothesis 1 (FOP labelling leads to more healthful choices), analyses compared FOP label groups (conditions 1-4; see Table 2) with the no-FOP label group (condition 5). To test Hypothesis 2 (MTL labels lead to more healthful choices than FuF labels), the MTL label groups (conditions 1 and 2) were compared with the FuF label groups (conditions 3 and 4). To test Hypothesis 3 (signage increases the healthfulness of food selections relative to FOP labels without signage), groups that included both FOP labels and signage (conditions 1 and 3) were compared with FOP label groups without signage (conditions 2 and 4).

Power analyses (two-tailed, $\alpha=0.05$ ) for ANOVA conducted to test Hypotheses 1-3 were performed using $\mathrm{G}^{*}$ Power ${ }^{(30)}$. These analyses suggested that all ANOVA had adequate power $(1-\beta=0.78)$ to detect moderate $(d=0.5)$, and large $(d=0 \cdot 8)$, effects $(1-\beta=0.99)$, but less than ideal power $(1-\beta=0 \cdot 17-0 \cdot 24)$ to detect small $(d=0 \cdot 2)$ effects.

In addition, MANOVA were used to examine whether main effects of FOP labelling, FOP label type and signage were moderated by factors identified in previous research as related to using nutrition information: participant motivation to purchase healthy food and demographic characteristics (parent and child BMI, family income and parental education) ${ }^{(31,32)}$.

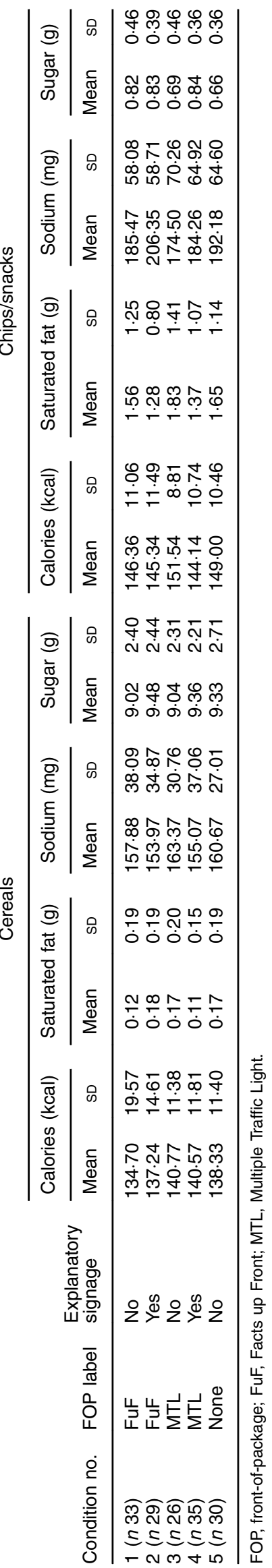




\section{Results}

Table 2 displays mean calories, fat, sodium and sugar per serving by product type and experimental condition.

Hypothesis 1, that FOP labelling would lead to more healthful food choices, was not supported. Although participants in the four FOP label groups ( $n$ 123) did select chips/snacks with marginally less sugar compared with those participants ( $n$ 30) without FOP labels (0.66 $v$. $\left.0.80 \mathrm{~g} ; \quad F(1,151)=2.95, P=0.088, \eta^{2}=0.02\right)$, on most comparison criteria (calories, saturated fat and sodium in all three categories, and sugar in cereals and crackers/ cookies) there were no significant differences based on FOP presence ( $P$ values ranged from 0.15 to $0 \cdot 98$ ). The only other $\eta^{2}$ value greater than 0.01 was $0.014(P=0 \cdot 15)$ for lower sodium in crackers/cookies among the FOP label group (149 mg) $v$. the control group (161 mg). See Table 3 for summary of study findings.

In addition, there was no evidence to support Hypothesis 2 that MTL labelling would lead to more healthful food choices compared with FuF labels. There were no significant differences for calories, saturated fat or sugar between the groups that selected foods from products labelled with MTL labels ( $n$ 61) $v$. FuF labels ( $n$ 62). For sodium $\left(F(1,121)=6 \cdot 07, P=0 \cdot 015, \eta^{2}=0 \cdot 05\right)$, those in the group exposed to FuF labels selected crackers/cookies with a lower average level of sodium (141 mg) compared with those exposed to MTL labels (158 mg). The FuF group also chose crackers/cookies with marginally fewer calories than the MTL group (136 v. $141 \mathrm{kcal}$ per serving; $\left.F(1,121)=3 \cdot 22, P=0 \cdot 075, \eta^{2}=0 \cdot 03\right)$. Among all other results, $P$ values ranged from 0.19 to 0.98 ; the only other $\eta^{2}$ value greater than 0.01 was $0.014(P=0 \cdot 19)$ for higher sodium in chips/snacks in FuF (195 mg) $v$. MTL (180 mg).

Finally, there was some evidence to support Hypothesis 3 that access to educational signage increases healthful food choices among participants who had access to FOP labels. These analyses included the 123 participants in conditions 1-4, where FOP labels were present. Compared with those without access to signage ( $n$ 59), those with access to signage ( $n$ 64) selected chips/snacks with fewer calories $(145 v .149 \mathrm{kcal} ; F(1,121)=4 \cdot 19, P=0 \cdot 043$, $\left.\eta^{2}=0.03\right)$ and marginally less saturated fat $(1.3 v .1 .7 \mathrm{~g}$; $\left.F(1,121)=2 \cdot 81, \quad P=0.096, \quad \eta^{2}=0.02\right)$, and marginally lower levels of saturated fat across all six selected products (1.0 v. $\left.1.2 \mathrm{~g} ; F(1,121)=3.62, P=0.059, \eta^{2}=0.03\right)$, but crackers/cookies with more sodium (156 v. $142 \mathrm{mg}$; $\left.F(1,121)=3.97, P=0.049, \eta^{2}=0.03\right)$. For non-significant results, $P$ values ranged from 0.16 to 0.99 , and $\eta^{2}$ values greater than 0.01 were for higher calories in cereals in the signage group (124v. $\left.120 \mathrm{kcal} ; \eta^{2}=0.012, P=0.24\right)$, higher sodium in chips/snacks in the signage group (194 v. $181 \mathrm{mg} ; \eta^{2}=0 \cdot 012, P=0 \cdot 23$ ) and higher sodium in all products in the signage group (168 v. $161 \mathrm{mg}$; $\left.\eta^{2}=0 \cdot 017, P=0 \cdot 16\right)$.
There were no significant interactions between participant motivation and label presence or signage presence, but there was a significant interaction between label type and motivation $\left(F(24,200)=1 \cdot 61, P=0.041, \eta^{2}=0 \cdot 16\right)$. Between-groups comparisons indicated that the only individual outcome for which this interaction was a significant predictor was sugar in chips/snacks $\left(F(2,110)=3 \cdot 46, P=0 \cdot 035, \eta^{2}=0 \cdot 06\right)$. Although this result reached statistical significance, its practical/clinical significance is dubious, as the mean sugar level for chips/ snacks purchased by all groups was less than $1 \mathrm{~g}$ (range $0.63-0.93 \mathrm{~g}$ ). In addition, there was a main effect of motivation $\left(F(24,260)=2 \cdot 49, P<0 \cdot 001, \eta^{2}=0 \cdot 19\right)$ on food healthfulness. This main effect was in the expected direction and was particularly evident for saturated fat in products chosen (i.e. less saturated fat in products chosen by individuals for whom it was 'very important' to purchase healthy food, followed by those for whom it was 'important', and the highest levels in products chosen by those for whom healthy purchasing was only 'somewhat important').

Parent BMI had a direct effect on food healthfulness such that parents with higher BMI selected cereals with fewer calories and chips/snacks with more calories and sodium than parents with lower BMI $(F(24,274)=1.63$, $\left.P=0.035, \eta^{2}=0 \cdot 13\right)$. However, parent BMI did not interact with label presence, label type or signage presence.

There were no main effects of child weight status (over 85th BMI percentile $v$. not), but child weight status did interact with in-aisle signage to predict food healthfulness $\left(F(12,108)=2 \cdot 09, P=0 \cdot 024, \eta^{2}=0 \cdot 19\right)$. Specifically, the presence of in-aisle signage influenced the sugar content of foods chosen by parent/child pairs in which the child's BMI was $>85$ th percentile, but the signage did not affect the sugar content of foods chosen by pairs with children having lower BMI. Among those pairs with a $>85$ th percentile BMI child, the impact of the signage on sugar content differed by product category, with signage predicting lower sugar among crackers/cookies ( $4.5 v .8 \mathrm{~g}$ for those without signage) but higher sugar among cereals (10.5 v. $8.5 \mathrm{~g}$ for no-signage pairs).

Neither parent education nor household income demonstrated direct or interactive effects on food healthfulness.

\section{Discussion}

Overall, the study hypotheses received little support. Although there were some results suggesting that FOP labelling and in-aisle explanatory signage were related to more healthful food choices, there were also findings suggesting that these FOP labels did not significantly contribute to more healthful choices. Given the forty-eight comparisons conducted across the study's three hypotheses and $\alpha$ level of 0.05 , one could expect to find 
approximately three significant results due to chance alone. Only three statistically significant results emerged across the three hypotheses, underscoring the conclusion that the FOP labelling systems tested here did not increase the healthfulness of food choices as anticipated.

Most counter to expectations, the multicoloured, intuitive MTL labels produced no more healthful food choices than the monochromatic FuF labels. Although many previous studies informed the hypothesis that consumers would make healthier choices with MTL labels than with FuF labels, not all previous research found traffic light labels to be superior to monochromatic labels. Grunert and colleagues reported that consumers were as able to understand products' healthfulness with traffic light labels as with monochromatic labels like $\mathrm{FuF}^{(33)}$. Similar results were reported by Malam and colleagues ${ }^{(28)}$.

The lack of MTL superiority over FuF labelling in the present study may owe, in part, to MTL labels' unfamiliarity to these participants, as MTL labels do not appear on foods in the USA. It is possible that, given the opportunity to use MTL labels, over time the hypothesized benefits of these labels would emerge among American consumers. Indeed, in other countries that have adopted traffic lightstyle labelling, consumers have reported liking, understanding and using these labels; and among FOP labelling systems, MTL labelling has accumulated the most research evidence of benefiting consumers in identifying more healthful products ${ }^{(22)}$.

Still, these labelling systems may be difficult for consumers to interpret. We found that there were few differences in nutrient composition of foods selected by those in the FOP label groups and those who saw no labels (control condition), especially before signage was added explaining how to use these labels, which may explain why FOP labels, even those that are colour coded with a familiar scheme (e.g. the red, amber and green colours of a traffic light), may not, on their own, convey clear nutrition messages to consumers. Moreover, the addition of explanatory in-aisle signage improved, to some extent, the healthfulness of foods chosen. Even the slight increase in sodium among cracker/cookie selections made by the participants with access to explanatory signage may be partially explained by these consumers attempting to make healthier choices. Choosing between savoury options (i.e. crackers) and sweet options (i.e. cookies), consumers seeking to make more healthful choices may have followed a heuristic strategy in which sugar was deemed the more important nutrient to reduce and/or cookies were considered less healthful than crackers despite the higher sodium content of the cracker options in this aisle. Tentative support for this position comes from a descriptive examination of the food choices made by participants with and without signage: $27 \%$ of participants without signage selected two sweet products (i.e. cookies) from the cracker/cookie aisle, whereas only $17 \%$ of the participants with signage did so. 
The somewhat increased healthfulness of products selected by participants in the signage conditions may be explained, in part, by the increased attention these participants paid to FOP labels. We previously reported that only one in four participants who had access to FOP labels, but no signage, actually viewed FOP labels, whereas nearly all participants in the signage conditions viewed the labels ${ }^{(10)}$. In order for any FOP labels to lead to more healthful dietary patterns, consumers must first view the labels, and our data indicate that in-aisle signage significantly increased the attention consumers paid to FOP labels. The value of attending to labels, particularly traffic light labels, for increasing healthy eating has also been recently demonstrated in a cafeteria setting ${ }^{(34)}$. Previous eye-tracking research also indicates that food package information to which consumers attend impacts their foodrelated decisions ${ }^{(35)}$. Thus, measures such as in-aisle signage that demonstrably increase attention to nutrition information should be considered key components of nutrition labelling programmes in order to promote consumer attention to the desired information.

Although it was expected that motivated consumers would make healthier choices when they had access to the FOP labels and that unmotivated consumers would not, the labels did not significantly impact motivated or unmotivated consumers' choices. Some previous research has also documented a lack of the interaction hypothesized here ${ }^{(36)}$; however, the previous results failed to support the interaction for essentially the opposite reason: use of nutrition information was linked with healthier consumption patterns among both motivated and unmotivated individuals.

Finally, consistent with previous research ${ }^{(37,38)}$, parent and child BMI were related to the healthfulness of foods selected. In the present study, main effects emerged for parent BMI, and interactions were demonstrated between child BMI and signage presence. Although the relationship between BMI and food choice was not uniform across product types (e.g. higher parent BMI was related to lower-calorie cereal choices and higher-calorie chip/snack selections), there was evidence that higher BMI was related to greater use of nutrition information. For example, the in-aisle explanatory signage influenced food choices among pairs with overweight, but not normal weight, children. This suggests that, encouragingly, the point-ofdecision information was guiding food choices made by pairs with overweight children.

\section{Strengths}

The current study was the first to compare the impact of FuF and MTL labels on consumers' food choices and also the first to examine the role played by explanatory signage in the FOP labelling/food choice context. A strength of this research was that participants actually took home the foods they selected, rather than just reporting purchase intentions. Collecting data regarding actual consumer food selections is beneficial because participants would be less likely to take home foods they did not actually want to eat, whereas participants seeking to convey an image of health-consciousness could self-report intending to purchase a healthy food if there were no real-world implications of stating such an intention (i.e. if they did not receive the chosen foods). Including children in the current study was another strength, as many food shopping trips are made by parents and children together and children frequently impact parents' food choices ${ }^{(39)}$. In addition, FOP labels have the potential to be understood and used by children and more readily explained to children by parents; therefore, food choices made by parents and children are of particular interest in studies of FOP labels.

\section{Limitations}

The study was designed to be similar to a real-life grocery shopping experience (i.e. products were located as they are in a grocery store, participants placed foods into a grocery basket, price tags were present) and to promote natural behaviour, participants were instructed to behave as they typically would when food shopping. It is possible that this instruction biased participants towards selecting foods they typically choose and reduced the potential impact of FOP labels and signage on food choices. It is also possible that characteristics of the study design (e.g. providing six foods - two from each of three categories free of charge) and laboratory setting produced some types of artificial behaviour and that these differences could represent important limitations of the present study.

In addition, providing participants with six foods to take home, rather than a fixed amount of money with which to select foods, may have led participants to choose items based on price rather than preference (i.e. participants may have sought to maximize value and selected the two most expensive items from each food category); however, this methodological choice was made deliberately to ensure an equal number of products were selected by each participant pair. In addition, this pattern of preferentially choosing more expensive foods was not observed in these data; for the three food categories, selected products averaged 1 cent more, 19 cents more and 6 cents less than the mean price of all available products in the category.

Requiring participants to choose two foods from each of the three categories also did not allow them to take advantage of the 'no-choice' option that they have in real life and may have influenced the choices made in the present study ${ }^{(40)}$. By not including the no-choice option, it was not possible for a participant to avoid a category of foods entirely or to opt for foods from a category that would be healthier overall than those available in the study (e.g. fresh produce). Although each category included in the study did contain products spanning a wide range of healthfulness (from low levels - green lights - for two or all three nutrients to limit to multiple red lights 
indicating high levels of these nutrients), it would be even more beneficial for public health if, in real life, FOP labels on packaged goods such as particularly unhealthy chips and snacks influenced consumers to select healthier unpackaged options, particularly fresh produce, rather than somewhat healthier chips and snacks. Due to study constraints, it was not feasible to stock the laboratory with fresh produce to examine this possibility.

In addition, the requirement to choose two foods from a category may have resulted in food choices interacting with one another ${ }^{(41)}$. For example, the attributes of one chosen food may have impacted subsequent choices through 'balancing', in which consumers who selected one unhealthy food then selected a healthier option to offset, or balance, the prior choice. In contrast, consumers might see an initial choice as 'highlighting' a salient goal (e.g. a healthier choice indicating that health goals are a priority, or a tastier but less healthy initial choice indicating that pleasure goals are a priority) and align subsequent food selections with the goal that received initial highlighting. Future research on the impact selected items have on one another (e.g. whether balancing or highlighting occurs within and/or between categories) would make an important contribution to understanding food choice.

It should also be noted that only two label types were examined in the current study. Although the MTL labels have been previously shown to be liked by and helpful for consumers $^{(22,42)}$, there is also evidence that simpler label formats (e.g. systems using stars or smiley faces) can be evaluated more quickly and easily by consumers ${ }^{(43)}$. Recent research suggests that evaluative labels, such as the 'Healthy Stars' initiative proposed by the Institute of Medicine, have a stronger impact on consumer behaviour than do reductive FOP labelling systems such as the FuF labels ${ }^{(44)}$. However, it should be noted that simpler, evaluative systems can mislead consumers to make more positive nutrient evaluations than are warranted ${ }^{(45)}$. In addition, although the present study did not test an exclusively evaluative system, the MTL labels provided both reductive (presenting only selected nutrients to limit) and evaluative (i.e. green, amber and red evaluations of nutrient healthfulness) elements. Nevertheless, it would be very informative for future research to compare additional FOP systems varying both on complexity and on reductive $v$. evaluative dimensions in regard to their impact on food choice. Finally, it should be noted that for any in-store nutrition labelling education efforts to succeed, supermarkets must be willing to provide this type of information; although it is unlikely that all grocers would be willing to devote space in their stores to nutrition education, there is evidence that many chains would do so. Many grocery chains (e.g. HyVee, King Soopers, Price Chopper, Tops, and at least twenty others ${ }^{(46)}$ ) have paid to license the NuVal labelling system and provide in-store signage explaining to consumers how to use this system.
Finally, power to detect small effects was limited in the current study. Therefore, although analyses suggest that we can have confidence that there are few moderateto-large (and to some extent small-to-moderate) differences in food choice based on the FOP labels tested here, small differences in food choice based on label exposure may have gone undetected. Although it is possible for small effects to accumulate across large groups and create meaningful population health benefits ${ }^{(47,48)}$, ideally, given the resources that would be required for implementation, an FOP system mandated by the Food and Drug Administration would produce effects on individual food choice that would be somewhat larger in magnitude.

\section{Future directions}

Prospective research would help clarify whether FOP labels with simplifying heuristic schemes, such as colour coding or symbols indicating varying levels of nutrients or overall product healthfulness, do provide advantages over FuF labels. It may take time for any new system to be recognized and used by consumers, but it is possible that once a system becomes familiar, those that are more intuitive or easier to use at a glance will be more helpful to consumers in identifying healthier choices. Previous eyetracking research reveals that consumers spend only about 1 second looking at nutrition information when making food choices ${ }^{(9,49)}$ and that FOP labels are viewed substantially more often than $\mathrm{NFL}^{(10)}$, likely due to their more prominent position on the front of a package and their ability to be viewed without having to remove a food package from a shelf; therefore, it is likely that an FOP label that can be viewed quickly and understood at a glance will be used more by consumers. The benefits of explanatory in-aisle signage that were previously reported with regard to visual attention ${ }^{(10)}$ and received some support in the present analysis with regard to food choice suggest that the introduction of any new FOP labelling system should be accompanied by an informational campaign to educate consumers about where to find, and how to use, the new labels. It would also be useful to examine how different forms of education provision (e.g. televised public service announcements $v$. in-aisle signage) affect consumer understanding and use of FOP labels.

\section{Conclusions}

Although the presence of FOP labels and explanatory signage predicted consumer selection of somewhat more healthful products, the benefits of FOP labels and signage were limited (i.e. benefits were seen only for certain nutrients in certain food categories) and were not as widespread as would be ideal for public health benefit. Although it is likely that consumers would make healthier food choices if a uniform FOP system was adopted and 
clearly explained, a different labelling system may be more beneficial for consumers than those tested here. Further, additional measures (e.g. increasing consumer knowledge of the benefits of reducing consumption of specific nutrients such as sodium and sugar, increasing consumer motivation for healthy eating, or promoting the good taste of healthier products) beyond merely adding new FOP labels may be necessary to increase the healthfulness of consumer choices.

\section{Acknowledgements}

Acknowledgements: The authors would like to acknowledge Brittany Niesen for her contributions to this study. Financial support: This work was supported by the Robert Wood Johnson Foundation's Healthy Eating Research Program (grant number 69295). The funder had no role in the design, analysis or writing of this article. Conflict of interest statement: None. Authorship: D.J.G. designed the study, conducted data analyses, and drafted the manuscript. R.G.L.-T. assisted with study design, data analysis, and critical revision of the manuscript. M.P.M. assisted with participant recruitment, conducting study visits, data management, and revising the manuscript. M.J. coordinated the study recruitment, conduct, and provided feedback on the manuscript. L.H. contributed to interpreting study findings and manuscript preparation. Ethics of buman subject participation: This study was conducted according to the guidelines laid down in the Declaration of Helsinki and all procedures involving human subjects were approved by the University of Minnesota Institutional Review Board. Written informed consent was obtained from all subjects.

\section{References}

1. Dietary Guidelines Advisory Committee (2010) Report of the Dietary Guidelines Advisory Committee on the Dietary Guidelines for Americans, 2010, to the Secretary of Agriculture and the Secretary of Health and Human Services. Beltsville, MD: US Department of Agriculture, Agricultural Research Service.

2. World Health Organization (2015) Healthy Diet, Fact Sheet No. 394. http://www.who.int/mediacentre/factsheets/fs394/ en/ (accessed September 2016).

3. Olshansky SJ, Passaro DJ, Hershow RC et al. (2005) A potential decline in life expectancy in the United States in the 21st century. New Eng J Med 352, 1138-1145.

4. Centers for Disease Control and Prevention (2013) Overweight and Obesity. http://www.cdc.gov/obesity/ data/childhood.html, (accessed August 2016).

5. Wang Y, Beydoun MA, Liang L et al. (2008) Will all Americans become overweight or obese? Estimating the progression and cost of the US obesity epidemic. Obesity (Silver Spring) 16, 2323-2330.

6. US Government, 101st Congress (1990) Nutrition Labeling Education Act of 1990. Public Law 101-535. https://www. congress.gov/bill/101st-congress/house-bill/3562 (accessed September 2016).
7. US Food and Drug Administration (2014) Proposed Changes to the Nutrition Facts Label. http://www.fda.gov/Food/ GuidanceRegulation/GuidanceDocumentsRegulatoryInforma tion/LabelingNutrition/ucm385663.htm (accessed February 2016).

8. European Food Information Council (2015) Global Update on Nutrition Labelling: Executive Summary. http://www. eufic.org/upl/1/default/doc/GlobalUpdateExecSumJan2015. pdf (accessed September 2016).

9. Graham DJ \& Jeffery RW (2011) Location, location, location: eye-tracking evidence that consumers preferentially view prominently positioned nutrition information. $J$ Am Diet Assoc 111, 1704-1711.

10. Graham DJ, Heidrick C \& Hodgin K (2015) Nutrition label viewing during a food-selection task: front-of-package labels vs nutrition facts labels. J Acad Nutr Diet 115, 1636-1646.

11. Campos S, Doxey J \& Hammond D (2011) Nutrition labels on pre-packaged foods: a systematic review. Public Health Nutr 14, 1496-1506.

12. Rothman RL, Housam R, Weiss H et al. (2006) Patient understanding of food labels: the role of literacy and numeracy. Am J Prev Med 31, 391-398.

13. Sinclair S, Hammond D \& Goodman S (2013) Sociodemographic differences in the comprehension of nutritional labels on food products. J Nutr Educ Behav 45, 767-772.

14. Cowburn G \& Stockley L (2005) Consumer understanding and use of nutrition labelling: a systematic review. Public Health Nutr $\mathbf{8}, 21-28$.

15. US Food and Drug Administration (2009) Background Information on Point of Purchase Labeling. http://www.fda. gov/Food/IngredientsPackagingLabeling/LabelingNutrition/ ucm187320.htm (accessed August 2016).

16. LetsMove (2013) Eat Healthy: Nutrition information. http:// www.letsmove.gov/eat-healthy (accessed August 2016).

17. Hersey JC, Wohlgenant KC, Arsenault JE et al. (2013) Effects of front-of-package and shelf nutrition labeling systems on consumers. Nutr Rev 71, 1-14.

18. Burton S \& Kees J (2012) Flies in the ointment? Addressing potential impediments to population-based health benefits of restaurant menu labeling initiatives. J Public Policy Mark 31, 232-239.

19. Andrews JC, Lin C-TJ, Levy AS et al. (2014) Consumer research needs from the food and drug administration on front-of-package nutritional labeling. J Public Policy Mark 33, $10-16$.

20. Grocery Manufacturers of America \& Food Marketing Institute (2011) Food and beverage industry launches Nutrition Keys front-of-pack nutrition labeling initiative to inform consumers and combat obesity. http://www. gmaonline.org/news-events/newsroom/food-and-beverageindustry-launches-nutrition-keys-front-of-pack-nutrition-/ (accessed August 2016).

21. UK Department of Health (2013) Guide to creating a front of pack (FoP) nutrition label for pre-packed products sold through retail outlets. https://www.gov.uk/government/ publications/front-of-pack-nutrition-labelling-guidance (accessed August 2016).

22. Hawley KL, Roberto CA, Bragg MA et al. (2013) The science on front-of-package food labels. Public Health Nutr 16, 430-439.

23. Balcombe K, Fraser I \& Di Falco S (2010) Traffic lights and food choice: a choice experiment examining the relationship between nutritional food labels and price. Food Policy 35, 211-220.

24. Borgmeier I \& Westenhoefer J (2009) Impact of different food label formats on healthiness evaluation and food choice of consumers: a randomized-controlled study. BMC Public Health 9, 184. 
25. Kelly B, Hughes C, Chapman K et al. (2009) Consumer testing of the acceptability and effectiveness of front-of-pack food labelling systems for the Australian grocery market. Health Promot Int 24, 120-129.

26. Roberto CA, Bragg MA, Schwartz MB et al. (2012) Facts up front versus traffic light food labels: a randomized controlled trial. Am I Prev Med 43, 134-141.

27. Roberto CA, Larsen PD, Agnew H et al. (2010) Evaluating the impact of menu labeling on food choices and intake. Am J Public Health 100, 312-318.

28. Malam S, Clegg S, Kirwan S et al. (2009) Comprehension and Use of UK Nutrition Signpost Labelling Schemes. London: Food Standards Agency.

29. Robert Wood Johnson Foundation Healthy Eating Research Program (2011) Using New Eye-Tracking Technologies to Assess the Effects of Varied Nutrition Labels on the Selection and Purchase of Healthful Foods. http://healthyeatingresearch. org/research/using-new-eye-tracking-technologies-to-assessthe-effects-of-varied-nutrition-labels-on-the-selection-andpurchase-of-healthful-foods/ (accessed August 2016).

30. Faul F, Erdfelder E, Lang A-G et al. (2007) G*Power 3: a flexible statistical power analysis program for the social, behavioral, and biomedical sciences. Behav Res Methods 39, 175-191.

31. Graham DJ \& Jeffery RW (2012) Predictors of nutrition label viewing during food purchase decision making: an eye tracking investigation. Public Health Nutr 15, 189-196.

32. Keller SB, Landry M, Olson J et al. (1997) The effects of nutrition package claims, nutrition facts panels, and motivation to process nutrition information on consumer product evaluations. J Public Policy Mark 16, 256-269.

33. Grunert KG, Wills JM \& Fernández-Celemín L (2010) Nutrition knowledge, and use and understanding of nutrition information on food labels among consumers in the UK. Appetite 55, 177-189.

34. Sonnenberg L, Gelsomin E \& Levy DE (2013) A traffic light food labeling intervention increases consumer awareness of health and healthy choices at the point-of-purchase. Prev Med 57, 253-257.

35. Bialkova S \& van Trijp HC (2011) An efficient methodology for assessing attention to and effect of nutrition information displayed front-of-pack. Food Qual Prefer 22, 592-601.

36. Graham DJ \& Laska MN (2012) Nutrition label use partially mediates the relationship between attitude toward healthy eating and overall dietary quality among college students. J Acad Nutr Diet 112, 414-418.

37. Halford JC, Boyland EJ, Hughes GM et al. (2008) Beyondbrand effect of television food advertisements on food choice in children: the effects of weight status. Public Health Nutr 11, 897-904.

38. Mela DJ (2001) Determinants of food choice: relationships with obesity and weight control. Obes Res 9, Suppl. 4, 249S-255S.

39. O'Dougherty M, Story M \& Stang J (2006) Observations of parent-child co-shoppers in supermarkets: children's involvement in food selections, parental yielding, and refusal strategies. J Nutr Educ Behav 38, 183-188.

40. Dhar R \& Simonson I (2003) The effect of forced choice on choice. J Mark Res 40, 146-160.

41. Dhar R \& Simonson I (1999) Making complementary choices in consumption episodes: highlighting versus balancing. J Mark Res 36, 29-44.

42. Roberto CA, Bragg MA, Seamans MJ et al. (2012) Evaluation of consumer understanding of different front-ofpackage nutrition labels, 2010-2011. Prev Chronic Dis 9, 120015.

43. Feunekes GI, Gortemaker IA, Willems AA et al. (2008) Front-of-pack nutrition labelling: testing effectiveness of different nutrition labelling formats front-of-pack in four European countries. Appetite 50, 57-70.

44. Newman CL, Howlett E \& Burton S (2014) Shopper response to front-of-package nutrition labeling programs: potential consumer and retail store benefits. J Retail 90, $13-26$.

45. Andrews JC, Burton S \& Kees J (2011) Is simpler always better? Consumer evaluations of front-of-package nutrition symbols. J Public Policy Mark 30, 175-190.

46. NuVal (2016) Where to find NuVal ${ }^{\circledR}$. https://www.nuval. com/location (accessed August 2016).

47. Rose G (1992) The Strategy of Preventive Medicine. Oxford: University Press.

48. Rose G (2001) Sick individuals and sick populations. Int J Epidemiology 30, 427-432.

49. Wolfson JA, Graham DJ \& Bleich SN (2016) Attention to physical activity-equivalent calorie information on Nutrition Facts Labels: an eye tracking investigation. J Nutr Educ Behav (In the Press). 